\title{
In-hospital and one-year mortality among patients with acute kidney injury and hematological malignancies
}

\author{
Inês Coelho ${ }^{1}$, Teresa Chuva ${ }^{2}$, Hugo Ferreira², Ana Paiva², José M. Costa ${ }^{2}$ \\ ${ }^{1}$ Hospital Amato Lusitano, Castelo Branco, Castelo Branco, Portugal \\ 2 Instituto Português de Oncologia do Porto Francisco Gentil EPE, Porto, Porto, Portugal
}

\section{ABSTRACT}

Hematological malignancies (HM) confer a high risk of acute kidney injury (AKI), which is associated with elevated morbi-mortality. The aim of this study was to identify the prognostic factors for in-hospital mortality and one-year mortality in this population. We conducted a single center, retrospective, observational cohort study of 101 in-hospital patients with AKI and HM between January 2015 and December 2019. Multiple myeloma was present in $30.7 \%$ of the patients, followed by non-Hodgkin lymphoma (NHL) in $27.7 \%$. Renal support therapy (RST) was needed in $60.4 \%$ of the cases. Independent predictors for in-hospital mortality were invasive mechanical ventilation (IMV) (OR 49.53; 95\% Cl:9.17 - 267.57; $\mathrm{P}<0.001$ ) and infection during in-hospital stay (IDHS) (OR 5.09; 95\% Cl:1.18 - 21.89; P=0.029). Predictors for one-year mortality were NHL (HR 2.88; 95\% Cl:1.54 - 5.39; P=0.001), tumor progression (HR 2.36; 95\% Cl:1.29 - 4.32; P=0.006) and IMV (HR 6.38; 95\% $\mathrm{Cl}: 3.50$ - 11.64; $\mathrm{P}<0.001)$. Higher albumin levels at AKI diagnosis conferred a better prognosis ( $\mathrm{HR} 0.57 ; 95 \% \mathrm{Cl}: 0.35-0.91 ; \mathrm{P}=0.020)$. Our model showed that patients with HM and AKI who were submitted to IMV and had IDHS had a probability of in-hospital death of $96 \%$. Albumin at the time of AKI influenced one-year mortality.

Keywords: Acute kidney injury; hematologic malignancies; hemodialysis; intensive care; prognosis; risk factors.

\section{INTRODUCTION}

Acute kidney injury (AKI) and electrolyte disturbances are the most common manifestations of kidney disease in patients with malignancy in a hospital setting and they are associated with high morbidity and mortality. Kidney failure alters target drug doses, compromising the success of anticancer treatments ${ }^{1}$. Renal disease also limits access to some types of therapies and excludes patients from clinical trials.

AKI incidence in these particularly vulnerable patients depends on the type of malignancy (solid tumor or hematological malignancies), severity of underlying disease, severity of complications and therapy. Depending on the definition of AKI, the incidence rate varies, but large population-based studies have supported that AKI is common in patients with cancer ${ }^{1,2}$. In one study that used a cut-off of $>50 \%$ increase in serum creatinine baseline level to define AKI, the rate of AKI was $17.5 \%$ within one year of cancer diagnosis ${ }^{2}$. According to Salahudeen et al. ${ }^{3}$ from University of Texas M.D. Anderson Cancer Center, AKI in-hospital was associated with a 4.7-fold increase in death rates, a 3-day increase in length of hospital stay, and a \$42.671 increase in hospital costs.

The cancers associated with the highest incidence of AKI in the ICU setting are hematological malignancies ${ }^{1}$. Among them, multiple myeloma (MM), non-Hodgkin lymphoma (NHL), acute myeloid leukemia (AML) and acute lymphoid leukemia (ALL) are the cancers with the highest risk of $\mathrm{AKI}^{4}$. Several factors may contribute to the risk of AKI in these patients, such as dehydration due to decrease oral intake, vomiting and diarrhea, fluid and electrolyte disturbances, intratubular and extrarenal urinary tract obstruction, tumor lysis syndrome (TLS), glomerulonephritis, sepsis or septic shock, nephrotoxicity from contrast administration, nonsteroidal anti-inflammatory drugs (NSAIDs), antibiotics and chemotherapy.

If we focus on the patients who develop AKI after hematopoietic stem cell transplantation (HSCT), the cause for AKI is often multifactorial and we can have more specific conditions such as marrow infusion syndrome, hepatic sinusoidal obstruction syndrome, thrombotic microangiopathy and graft versus host disease (GVHD) ${ }^{5}$. Studies demonstrated remarkably high rates of AKI after myeloablative allogeneic transplantation, reaching $73 \%$ and a four-fold greater need for dialysis compared with patients in the nonmyeloablative group ${ }^{6,7}$. Unfortunately, patients requiring acute dialysis have extremely high (80\%) mortality rates 5 and the best management of these population remains a challenging task.

Our aim was to search for the prognostic determinants for in-hospital mortality and one-year mortality in patients with HM and AKI.

\section{MATERIALS AND METHODS}

\section{Patients and Data Collection}

We conducted a single-center, retrospective, observational cohort study of 101 in-hospital patients with AKI and HM between January 2015 and December 2019. Our center is a tertiary referral oncological 
center. Essential demographic, clinical and laboratory data were recorded from medical records at baseline, time of AKI event, 1 and 12 months after AKI.

Patients had primary diagnosed HM at different stages: partial or complete remission, as well as disease progression.

Data collection included basic demographic details, comorbidities, type of HM, history of solid malignancies, neoplasia status, treatment with stem cell transplantation (SCT), admission to the ICU, need for invasive mechanical ventilation (IMV), presence of septic shock, graftversus-host disease (GVHD), characterization of AKI (exclusive pre-renal, obstructive, nephrotoxicity, hypercalcemia, tumor lysis syndrome (TLS), glomerulonephritis, thrombotic microangiopathy, tumor infiltration), need for renal support therapy (RST) and modality of RST. Serum creatinine levels were recorded at baseline, time of AKI event, 1 and 12 months after AKI, and albumin was recorded at the time of AKI event.

Renal function was assessed according to serum creatinine concentration and estimated glomerular filtration rate (GFR) by the Modified Diet in Renal Disease equation (MDRD). Baseline serum creatinine was the lowest representative value determined from several measurements available in the last 1-2 months before hospital admission. Baseline renal function was classified into 5 stages, as suggested by the National Kidney Foundation (NKF). We classified AKI according to the KDIGO definition. Urine output was not available for all patients, so the serum creatinine was the only parameter we used.

For the patients admitted in the ICU, two mortality prediction scores were calculated: Acute Physiology and Chronic Health Evaluation (APACHE II) and Simplified Acute Physiology Score (SAPS II).

We defined infection during in-hospital stay as Systemic Inflammatory Response Syndrome (SIRS), sepsis or septic shock.

Patients for whom palliative care was the only cancer treatment available were excluded. Clinical outcomes were assessed as in-hospital death and death at one year.

The ethics committee of our Institution approved the study, including the fact that informed consent was not sought due to a retrospective study design (approval No.: 229/020 issued on 23 July 2020).

\section{Statistical analysis and prediction model}

Discrete variables were summarized by absolute and relative frequencies. Continuous variables were summarized using measures of central tendency and dispersion, mean, standard deviation (SD), median and interquartile range (IQR).

Univariate analyses were performed using Student's t-test or MannWhitney $\mathrm{U}$ tests for continuous variables, and the chi-square test or Fisher's exact test for categorical variables, as appropriate. GFR comparisons were performed by Friedman test.

A multivariate logistic regression analysis was performed to assess in-hospital mortality. Demographic and clinical variables were tested as in-hospital mortality predictors using Stepwise forward method, considering the likelihood ratio (LR) test. For each variable in the final model, adjusted odds ratio, and respective $95 \%$ confidence interval were estimated. The goodness-of-fit was assessed by calculating the area under the receiver operating characteristic (ROC) curve, the concordance index (C-statistic), and applying the Hosmer-Lemeshow test. ROC curve value $>0.7$ was considered to indicate predictive accuracy.

The Kaplan-Meier method was used to estimate the survival function for the time since hospital admission until death. The log-rank test was used to compare survival between groups.

Cox proportional hazards models were fitted to assess predictors of one-year mortality. The same variables were tested as those in logistic regression, and Stepwise forward method with LR test was also used for each variable in the final model. Adjusted hazard ratios, and respective $95 \%$ confidence interval, were estimated.

There were no missing values. A $p$-value $<0.05$ was considered statistically significant. Statistical analyses were performed using SPSS statistical program (IBM SPSS 19.0, Chicago, IL, USA).

\section{RESULTS}

\section{Patients' characteristics}

The study population included 64 males and 37 females, with a mean age of $58.7 \pm 16.8$ years. Only one patient was African-American; all the others were Caucasian. Multiple myeloma was present in 31 $(30.7 \%)$ patients, followed by non-Hodgkin lymphoma (LNH) in 28 (27.7\%). Fourteen (13.9\%) patients had acute myeloid leukemia (AML) and $9(8.9 \%)$ had acute lymphoid leukemia (ALL). The remaining diagnoses (19 (18.8\%) patients) included other hematological diseases such as Waldenström macroglobulinemia, myelofibrosis, myelodysplastic syndrome and aplastic anemia. Thirty-eight (37.6\%) patients needed SCT and 27 of them underwent allogenic transplantation.

Fifty-two patients (51.5\%) were admitted to intensive care unit (ICU) and sixty-one (60.4\%) needed renal support therapy (RST). Mean length of in-hospital stay was 18 days (IQR 1-88). In-hospital death was $52.5 \%$ and after one year only 26 patients were alive. The baseline characteristics are listed in Table I.

The demographic, clinical and laboratorial data were compared between survivors and in-hospital death patients. The univariate analysis identified significant predictive factors for in-hospital mortality: infection during hospital stay $(P<0.001)$, underlying $\mathrm{HM}(\mathrm{P}<$ $0.001)$, stem cell transplantation $(P=0.001)$, graft-versus-host disease $(P=0.021)$, AKI KDIGO stage $3(P=0.020)$, nephrotoxicity $(P<0.001)$, admission to ICU $(P<0.001)$, SAPS II score $(P<0.001)$, IMV $(P<0.001)$, septic shock $(P<0.001)$, and RST $(P<0.001)$.

Factors that were protective against in-hospital death were: age $(P<0.001)$, history of hypertension $(P=0.004)$, higher albumin at AKI diagnosis $(P<0.001)$, exclusive pre-renal AKI $(P=0.002)$ and hypercalcemia $(P=0.013)$. 


\section{Table I}

Patient characteristics with and without in-hospital death.

\begin{tabular}{|c|c|c|c|c|}
\hline Characteristic & $\begin{array}{c}\text { Survivors } \\
(n=48)\end{array}$ & $\begin{array}{l}\text { In-hospital death } \\
\qquad(\mathrm{n}=53)\end{array}$ & P-value & $\begin{array}{c}\text { Overall } \\
(N=101)\end{array}$ \\
\hline \multicolumn{5}{|l|}{ Gender } \\
\hline Female & $20(54.1)$ & $17(45.9)$ & \multirow{2}{*}{0.318} & $37(36.6)$ \\
\hline Male & $28(43.8)$ & $36(56.3)$ & & $64(63.4)$ \\
\hline Age (years) & $65.35 \pm 13.57$ & $52.64 \pm 17.34$ & $<0.001$ & $58.68 \pm 16.84$ \\
\hline Age $<60$ years & $13(29.5)$ & $31(70.5)$ & 0.001 & $44(43.6)$ \\
\hline History of hypertension & $31(62)$ & $19(38)$ & 0.004 & $50(49.5)$ \\
\hline Diabetes mellitus & $12(44.4)$ & $15(55.6)$ & 0.708 & $27(26.7)$ \\
\hline Cardio or cerebrovascular disease & $16(61.5)$ & $10(38.5)$ & 0.097 & $26(25.7)$ \\
\hline Obesity & $8(57.1)$ & $6(42.9)$ & 0.437 & $14(13.9)$ \\
\hline History of solid tumors & $9(60)$ & $6(40)$ & 0.294 & $15(14.9)$ \\
\hline Albumin at AKI diagnosis & $3 \pm 0.7$ & $2.5 \pm 0.5$ & $<0.001$ & $2.8 \pm 0.6$ \\
\hline Infection during in-hospital stay & $14(22.2)$ & $49(77.8)$ & $<0.001$ & $63(62.4)$ \\
\hline \multicolumn{5}{|l|}{ Underlying HM } \\
\hline$A M L$ & $3(21.4)$ & $11(78.6)$ & \multirow{5}{*}{$<0.001$} & $14(13.9)$ \\
\hline ALL & $1(11.1)$ & $8(88.9)$ & & $9(8.9)$ \\
\hline NHL & $11(39.3)$ & $17(60.7)$ & & $28(27.7)$ \\
\hline $\mathrm{MM}$ & $27(87.1)$ & $4(12.9)$ & & $31(30.7)$ \\
\hline Others & $6(31.6)$ & $13(68.4)$ & & $19(18.8)$ \\
\hline \multicolumn{5}{|l|}{ HM status } \\
\hline Diagnosis/Induction therapy & $21(56.8)$ & $16(43.2)$ & \multirow{3}{*}{0.168} & $37(36.6)$ \\
\hline Remission (partial or complete) & $11(34.4)$ & $21(65.6)$ & & $32(31.7)$ \\
\hline Progression & $16(50)$ & $16(50)$ & & $32(31.7)$ \\
\hline \multicolumn{5}{|l|}{ Stem cell transplantation } \\
\hline No & $38(60.3)$ & $25(39.7)$ & \multirow{3}{*}{0.001} & $63(62.4)$ \\
\hline Allo & $5(18.5)$ & $22(81.5)$ & & $27(26.7)$ \\
\hline Auto & $5(45.5)$ & $6(54.5)$ & & $11(10.9)$ \\
\hline Graft-versus-host disease & $3(20)$ & $12(80)$ & 0.021 & $15(14.9)$ \\
\hline \multicolumn{5}{|l|}{ AKI KDIGO stages } \\
\hline Stage 1 & $3(75)$ & $1(25)$ & \multirow{3}{*}{0.020} & $4(4)$ \\
\hline Stage 2 & $7(87.5)$ & $1(12.5)$ & & $8(7.9)$ \\
\hline Stage 3 & $38(42.7)$ & $51(57.3)$ & & $89(88.1)$ \\
\hline \multicolumn{5}{|l|}{ AKI etiology } \\
\hline Exclusive prerenal & $10(90.9)$ & $1(9.1)$ & 0.002 & $11(10.9)$ \\
\hline Obstructive & $5(71.4)$ & $2(28.6)$ & 0.252 & $7(6.9)$ \\
\hline Nephrotoxicity & $24(35.3)$ & $44(64.7)$ & $<0.001$ & $68(67.3)$ \\
\hline Tumor lysis syndrome & $4(40)$ & $6(60)$ & 0.744 & $10(9.9)$ \\
\hline Hypercalcemia & $8(88.9)$ & $1(11.1)$ & 0.013 & $9(8.9)$ \\
\hline Glomerulonephritis & $2(100)$ & $0(0)$ & 0.223 & $2(2)$ \\
\hline Thrombotic microangiopathy & $0(0)$ & $2(100)$ & 0.496 & $2(2)$ \\
\hline Tumor renal infiltration & $5(62.5)$ & $3(37.5)$ & 0.472 & $8(7.9)$ \\
\hline Intensive care unit & $8(15.4)$ & $44(84.6)$ & $<0.001$ & $52(51.5)$ \\
\hline Score APACHE II & $25 \pm 7$ & $32 \pm 9$ & 0.051 & $30.7 \pm 8.9$ \\
\hline Score SAPS II & $50 \pm 9$ & $72 \pm 22$ & $<0.001$ & $68.4 \pm 21.9$ \\
\hline Invasive mechanical ventilation & $2(4.3)$ & $44(95.7)$ & $<0.001$ & $46(45.5)$ \\
\hline Septic shock & $3(6.7)$ & $42(93.3)$ & $<0.001$ & $45(44.6)$ \\
\hline Renal support therapy & $18(29.5)$ & $43(70.5)$ & $<0.001$ & $61(60.4)$ \\
\hline \multicolumn{5}{|l|}{ Renal support therapy modality } \\
\hline No & $30(75)$ & $10(25)$ & & $40(39.6)$ \\
\hline HD & $15(75)$ & $5(25)$ & $<0.001$ & $20(19.8)$ \\
\hline CVVH & $2(6.5)$ & $29(93.5)$ & & $31(30.7)$ \\
\hline $\mathrm{HD}$ and $\mathrm{CVVH}$ & $1(10)$ & $9(90)$ & & $10(9.9)$ \\
\hline
\end{tabular}

Data are shown as mean \pm standard deviation (SD) or $n(\%)$. p-Values below 0.05 were marked as bold. HM, hematological malignancies; HD, hemodialysis; CVVH, continuous venovenous hemofiltration; AML, acute myeloid leukemia; ALL, acute lymphoid leukemia; NHL, non-Hodgkin lymphoma; MM, multiple myeloma. 


\section{Figure 1}

(a) Comparison between basal, one-month and one-year GFR; $N=17$. (b) Comparison between basal and one-month GFR; $N=40$.

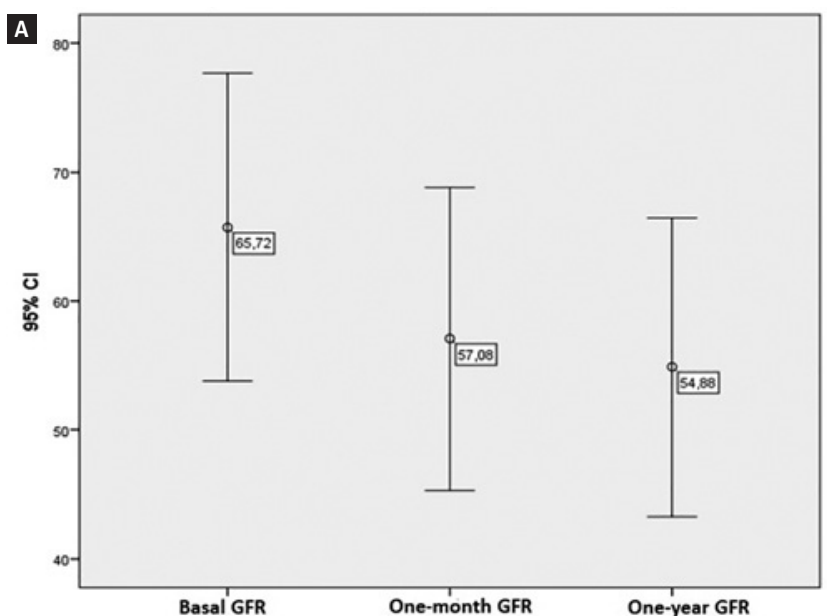

\section{GFR comparison}

Seventeen patients had registers of GFR at 3 moments: basal, onemonth and one-year. Basal GFR, one-month GFR and one-year GFR were, respectively, $65.7 \pm 28.9 \mathrm{~mL} / \mathrm{min} / 1.73 \mathrm{~m}^{2}, 57.1 \pm 28.5 \mathrm{~mL} /$ $\mathrm{min} / 1.73 \mathrm{~m}^{2}$ and $54.9 \pm 28.1 \mathrm{~mL} / \mathrm{min} / 1.73 \mathrm{~m}^{2}$. There were statistically significant differences between the values of basal GFR and one-month GFR $(P=0.033)$, and basal GFR and one-year GFR $(P=0.017) .40$ patients had data on both basal GFR and one-month GFR: $60.6 \pm 28.9$ $\mathrm{mL} / \mathrm{min} / 1.73 \mathrm{~m}^{2}$ and $51.2 \pm 29.2 \mathrm{~mL} / \mathrm{min} / 1.73 \mathrm{~m}^{2}(\mathrm{P}=0.003)$, respectively. These results are shown in Figure 1.

\section{Predictors of mortality}

The independent predictors for in-hospital mortality are listed in Table II.

\section{Table II}

Multivariate logistic regression analysis of the predictors for in-hospital death.

\begin{tabular}{l|c|c|c}
\multicolumn{1}{c|}{ Predictors } & OR & 95\% Cl & P-value \\
\hline IMV & 49.526 & $9.167-267.567$ & $<0.001$ \\
Infection during in-hospital stay & 5.091 & $1.184-21.887$ & 0.029
\end{tabular}

IMV, invasive mechanical ventilation.

The $\beta$ coefficient of the two variables were used to estimate the probability of in-hospital death according to the equation:

$$
P=\frac{1}{1+e^{\beta}} \text { with } \beta=-2.365+3.903 \times I M V+1.628 \times I D H S
$$

IMV, invasive mechanical ventilation; IDHS, infection during inhospital stay.

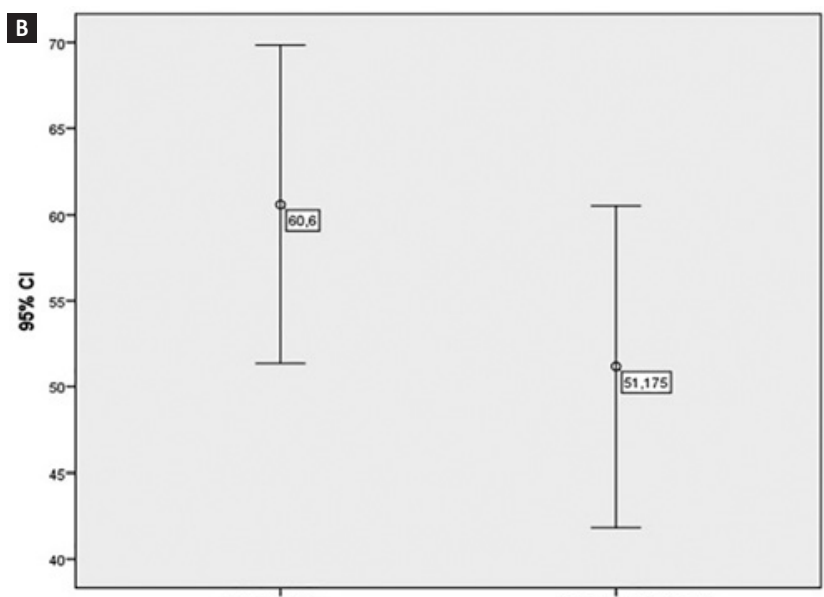

Basal GFR

One-month GFR

The probability of in-hospital death for a given risk factor combination, based on the equation model, is shown in Table III.

\section{Table III}

Probability of in-hospital death according to each of the two risk factors.

\begin{tabular}{c|c|c} 
IMV & IDHS & Probability of in-hospital death, $\%$ \\
\hline No & No & 8.6 \\
No & Yes & 32.4 \\
Yes & No & 82.3 \\
Yes & Yes & 96
\end{tabular}

IMV, invasive mechanical ventilation; IDHS, infection during in-hospital stay.

For a patient with IMV and infection during hospital stay, the probability of in-hospital death was $96 \%$.

According to the Hosmer-Lemeshow test, the reliability of the models was adequate $(P=0.882)$. Figure 2 represents the ROC curve drawn for the predicted probabilities of the logistic regression model. The C-statistic was 0.927 (95\% $\mathrm{Cl} 0.871-0.983)$, indicating that the equation with two variables had great discriminatory power. Based on the Youden index-optimized cut-off value of 0.573 (probability of in-hospital death $57.3 \%$ ), the model had a sensitivity of $83 \%$ and a specificity of $95.8 \%$.

\section{$\underline{\text { Table IV }}$}

Independent predictors for one-year mortality (Cox proportional hazard model).

\begin{tabular}{l|c|c|c}
\multicolumn{1}{c|}{ Predictors } & HR & 95\% Cl & P-value \\
\hline NHL & 2.883 & $1.542-5.391$ & 0.001 \\
Tumor progression & 2.356 & $1.286-4.315$ & 0.006 \\
IMV & 6.383 & $3.502-11.635$ & $<0.001$ \\
Albumin & 0.567 & $0.351-0.914$ & 0.020
\end{tabular}

$\mathrm{NHL}$, non-Hodgkin lymphoma; IMV, invasive mechanical ventilation. 


\section{Figure 2}

ROC curve to predict in-hospital death in our study population. Sensitivity: $83 \%$; Specificity: 95.8\%; C-statistic: 0.927; $P<0.001$.

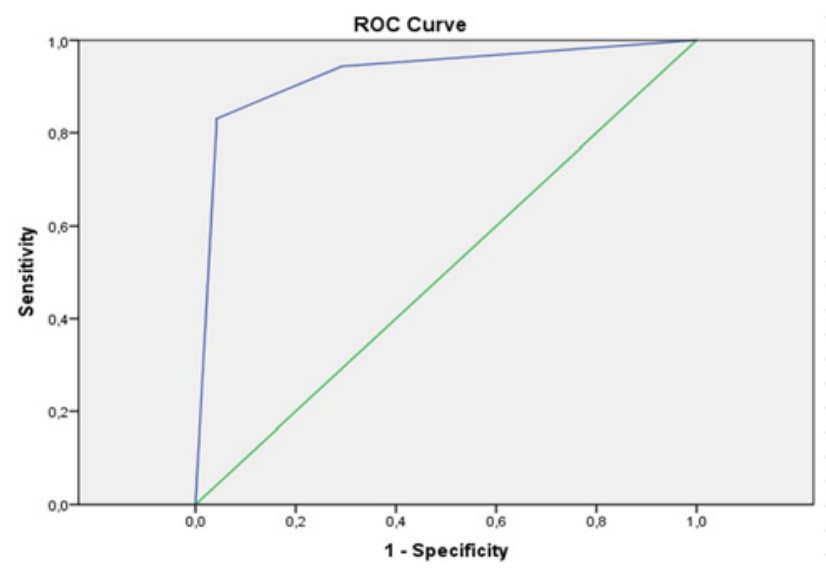

The independent predictors for one-year mortality are listed in Table IV.

\section{Survival curves}

The cumulative survival is represented in Figure 3. Survival rates were $65.3 \%, 35.6 \%, 24.8 \%, 20 \%, 20 \%$ and $17.8 \%$ at 1 month, 6 months, 1, 2, 3 and 4 years, respectively.

\section{Figure 3}

Kaplan-Meier mortality curve.

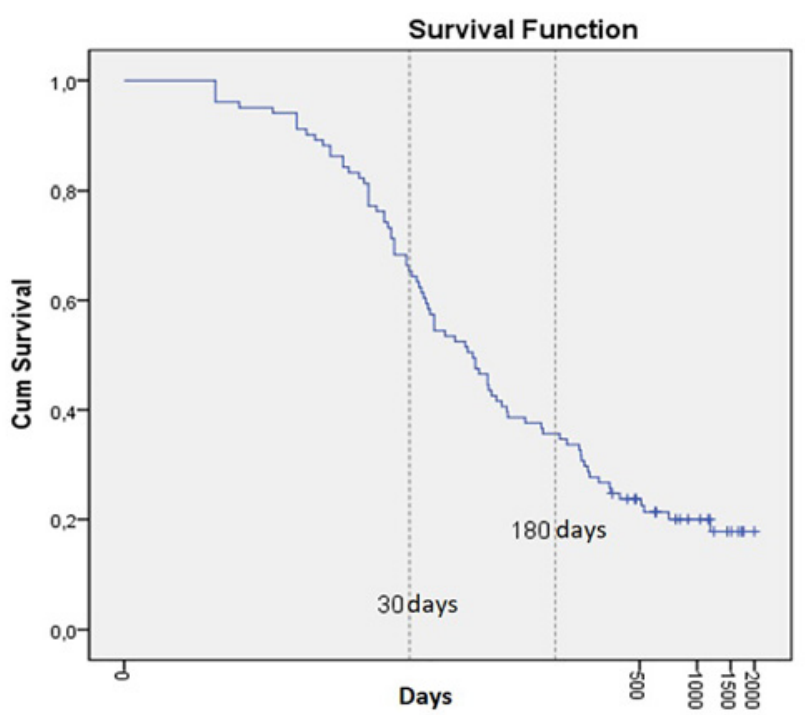

\section{DISCUSSION}

Patients with $\mathrm{HM}$ are at the highest risk for AKI development in most case series. AKI may be due to the direct effects of the malignancy, such as light chain cast nephropathy in patients with multiple myeloma, or may be a consequence of the therapy, that leads to immunosuppression and neutropenia, making patients more susceptible to sepsis ${ }^{8}$. AKI etiologies in patients with cancer have become more complex and multi-factorial. Clinicians should focus more on AKI prevention, as AKI has the potential to significantly worsen outcomes, particularly among patients with $\mathrm{HM}^{9}$.

Our work aimed to determine which prognostic features contribute to in-hospital and one-year mortality of patients with HM and AKI. There is a lack of studies in this particular population. We built a predictive model to assess the prognosis, which could help in the clinical decision-making process.

We found that HM patients who developed AKI were more frequently male $(63.4 \%)$, with a mean age of $58.68 \pm 16.84$ years and had MM (30.7\%). Our population is older and the incidence of MM is higher than in previous studies ${ }^{1,4}$.

Among the etiologies of AKI, nephrotoxicity was the most prevalent (67.3\%). This finding is supported by previous studies ${ }^{10,11}$. It was mainly associated with antibiotics (vancomycin, aminoglycosides, antifungals and antivirals) and less often to chemotherapy drugs. Radiocontrast-associated nephropathy is overestimated, since today we use hypoosmolar media and actively apply intravenous hydration to prevent contrast nephropathy in patients with serum creatinine $>1.5 \mathrm{mg} / \mathrm{dL}$ or GFR<60mL/min $/ 1.73 \mathrm{~m}^{2}$ with comorbidities or significant proteinuria, or GFR $<45 \mathrm{~mL} / \mathrm{min} / 1.73 \mathrm{~m}^{2}$ even in the absence of proteinuria or any other comorbidities. The study of Wilhelm-Leen E. et al. ${ }^{12}$ suggests the same. In the cases where contrast medium could have a role in the physiopathology of AKI, the patients were also treated with a variety of other nephrotoxic medications, mainly antimicrobial agents due to septic complications or were also exposed to calcineurin inhibitors (CNI).

TLS appeared in few cases (9.9\%), probably due to the extensive use of prophylactic rasburicase and allopurinol in our institution, which is an effective preventive measure ${ }^{13}$ Tumor infiltration is a common, but under-recognized complication of $\mathrm{HM}$ and was diagnosed in $7.9 \%$ of the patients. Thrombotic microangiopathy $(2 \%)$ was seen in allogeneic HSCT patients. Glomerulonephritis (2\%) corresponded to a case of light-chain deposition disease and a case of ANCA vasculitis with tumor infiltration by NHL. We had 3 cases of hemophagocytic lymphohistiocytosis (HLN), which has been reported in association with malignancies, most commonly lymphoid cancers (including B, T, and NK cell) and leukemias. Importantly, some aggressive malignancies may manifest as sepsis-like syndromes with multiple organ failures, comprehending HLH or severe TLS and emergent chemotherapy treatment is started in patients receiving life-sustaining therapies ${ }^{14}$.

AKI occurs in $~ 50 \%$ of patients after HSCT and is one of the most common serious complications of $\mathrm{HSCT}^{15}$. The main causes of AKI in this particular population where the association of sepsis, septic shock with ischemic insults and nephrotoxic drugs like CNI and antibiotics. 
Typically, this occurs within 30 days of the transplant and early-onset AKI is a poor prognostic factor for allogeneic HSCT recipients ${ }^{16}$. Thrombotic microangiopathy resulted from drugs, namely calcineurin inhibitors and acute GVHD. Total body irradiation, which is often related to thrombotic microangiopathy, is not performed at our institution. The most common forms of GVHD were cutaneous and gastrointestinal, which results in severe diarrhea contributing to prerenal AKI. Antivirals prophylaxis or therapeutics lead to tubular injury. In our population there were 2 cases of sinusoidal obstruction syndrome that initiated as a prerenal $\mathrm{AKI}$ and lately evolved to acute tubular injury. Autologous HSCT is associated with a relatively lower incidence of AKI, when compared to allogenic HSCT, probably because patients do not suffer from graft versus host disease (GVHD) and do not require the use of calcineurin inhibitors ${ }^{17}$.

GFR comparisons corroborated that AKI in cancer patients is associated with a higher risk of developing chronic kidney disease (CKD) ${ }^{9}$. In our population, the mean basal GFR was $>60 \mathrm{~mL} / \mathrm{min} / 1.73 \mathrm{~m}^{2}$, and after an AKI event, the mean one-month and one-year GFR corresponded to CKD stage G3a.

Our findings revealed that the association of IMV and infection during hospital stay yielded a $96 \%$ risk of in-hospital death. Chuva T. et al. ${ }^{4}$ reported, in a study conducted at our institution between 1995 and 2014, that septic shock, IMV and allogeneic SCT were independent predictors of death in this population, corroborating our results and showing that infection, independent of ICU admission and IMV, had a huge burden in the prognosis of these patients and that these findings have not changed in recent years, despite therapeutic advances. The independent predictors for one-year mortality were NHL, tumor progression and IMV. Indeed, the need for IMV has been associated with mortality in numerous studies ${ }^{18,19}$.

We found that more elevated levels of albumin at AKI diagnosis conferred a better prognosis at one year, probably not only reflecting the severity of infection during in-hospital stay as it is a negative acute-phase reagent of inflammation, but also representing the nutritional status of the patients who predicts its survival at one year. Lahoti et al. ${ }^{20}$ highlighted the presence of hypoalbuminemia as a significant predictor of AKI.

There is a lack of data about timing, modality and dose of RST in the setting of patients with malignancy and AKI, and ethical concerns about initiation of RST in these patients, make decisions very difficult for the nephrologist. In our study, $51.5 \%$ of the patients were observed in the ICU setting and overall mortality was $52.5 \%$. Higher mortality rates are reported in the studies conducted only in ICU patients ${ }^{10,21}$, and if the series include allogeneic HSCT recipients, the survival is even lower. These ICU-admitted patients developing AKI are much sicker patients, needing RST not only for uremia but also for inadequate water balance, and it is not related with the RST per se. However, Darmon et al. ${ }^{10}$ concluded that in-hospital mortality of HM patients developing AKI or requiring RST is close to the general ICU population, and Azoulay et al. ${ }^{22}$ affirms that over the last two decades, the number of patients with HM admitted to the ICU has increased and their mortality has dropped sharply. In our study the overall survival rates were $65.3 \%, 35.6 \%, 24.8 \%, 20 \%, 20 \%$ and $17.8 \%$ at 1 month, 6 months, $1,2,3$ and 4 years, respectively.
In our population, $60.4 \%$ of the patients needed RST: $30.7 \%$ CVVH, $19.8 \%$ HD, 9.9\% CVVH and HD. The choice of RST technique depended mainly upon hemodynamics, degree of impairment of other organs, modalities available in our unit and trained staff.

Our study has a few limitations. First, our report is limited by a retrospective single-center design, which limits the generalization of our findings to other institutions or patient populations. Second, we included a broad spectrum of hematological diagnosis with different diseases that have per se different clinical characteristics. For example, ALL affects younger patients, and MM has a higher incidence in older patients, which could limit comparisons. Third, many patients were exposed to more than one factor that could contribute to the etiology and physiopathology of AKI, so it's difficult to assess the real contribution of each factor and consequently its burden on the prognosis.

\section{CONCLUSIONS}

Our model showed that HM patients with AKI are at high risk of IDHS and IMV, resulting in elevated in-hospital death. Elevated levels of albumin at AKI diagnosis correlated with a better one-year survival, while NHL, cancer progression and IMV were risk factors for death.

Although RST in this population is linked with high mortality rates, mainly in patients admitted to ICU, this is related with the disease severity and not with the RST per se. Therapy should be individualized. Further multicentre research is needed in this particular population that will allow validation of our model and will create tools to help in the clinical decision-making process.

Acknowledgments: We thank Dr. Adriana Belo, MsC in Biostatistics, Department of Statistics and Operational Research, University of Lisbon, for performing statistical analysis.

Ethics approval: The ethics committee of Instituto Português de Oncologia do Porto Francisco Gentil EPE approved the study.

Disclosure of potential conflicts of interest: none declared.

\section{References}

1. Canet E, Zafrani L, Lambert J, Thieblemont C, Galicier L, Schnell D, Raffoux E, Lengline E, Chevret $S$, Darmon M, Azoulay E. Acute kidney injury in patients with newly diagnosed high-grade hematological malignancies: impact on remission and survival. PLoS One. 2013; 8(2):e55870. DOI: 10.1371/journal.pone.0055870

2. Christiansen CF, Johansen MB, Langeberg WJ, Fryzek JP, Sørensen HT. Incidence of acute kidney injury in cancer patients: a Danish population-based cohort study. Eur J Intern Med. 2011; 22:399-406. DOI: 10.1016/j.ejim.2011.05.005.

3. Salahudeen AK, Doshi SM, Pawar T, Nowshad G, Lahoti A, Shah P. Incidence rate, clinical correlates, and outcomes of AKI in patients admitted to a comprehensive cancer center. Clin J Am Soc Nephrol. 2013; 8(3):347-354. DOI:10.2215/CJN.03530412.

4. Chuva T, Maximino J, Barbosa J, Silva S, Paiva A, Baldaia J, Loureiro A. Haematological malignancies and acute kidney injury requiring nephrology consultation: challenging the worst of the worst. Clin Kidney J. 2016; 9(3):418 -423. DOl:10.1093/ckj/sfw026.

5. Renaghan AD, Jaimes EA, Malyszko J, Perazella MA, Sprangers B, Rosner MH. Acute kidney injury and CKD associated with hematopoietic stem cell transplantation. CJASN. 2020; 15(2):289-297. DOI: https://doi.org/10.2215/CJN.08580719.

6. Parikh CR, Schrier RW, Storer B, Diaconescu R, Sorror ML, Maris MB, Maloney DG, McSweeney $\mathrm{P}$, Storb R, Sandmaier BM. Comparison of ARF after myeloablative and nonmyeloablative 
hematopoietic cell transplantation. Am J Kidney Dis. 2005; 45:502-509. DOI: 10.1053/j.ajkd. 2004.11.013.

7. Lopes JA, Jorge S, Silva S, de Almeida E, Abreu F, Martins C, do Carmo JA, Lacerda JF, Prata MM. Acute renal failure following myeloablative autologous and allogeneic hematopoietic cell transplantation. Bone Marrow Transplant. 2006; 38:707. DOI: 10.1038/sj.bmt.1705513.

8. Canet E, Vincent F, Darmon M, Soares M. Acute kidney injury in hematological patients. Curr Opin Crit Care. 2015; 21:549-558. DOI: 10.1097/MCC.0000000000000253.

9. Rosner MH, Perazella MA. Acute kidney injury in the patient with cancer. Kidney Res Clin Pract. 2019; 38(3):295-308. doi:10.23876/j.krcp.19.042

10. Darmon M, Vincent $F$, Canet $E$, Mokart, Pène $F$, Kouatchet $A$, Mayaux J, Nyunga $M$, Bruneel $F$ Rabbat A, Lebert C, Perez P, Renault A, Meert A.P, Benoit D, Hamidfar R, Jourdain M, Schlemmer $B$, Chevret S, Lemiale $V$ and Azoulay E. Acute kidney injury in critically ill patients with haematological malignancies: results of a multicentre cohort study from the Groupe de Recherche en Réanimation Respiratoire en OncoHématologie. Nephrol Dial Transplant. 2015. 30:2006-2013. DOI: 10.1093/ndt/gfv372.

11. Harris KP, Hattersley JM, Feehally J, Walls J. Acute renal failure associated with haematological malignancies: a review of 10 years experience. Eur J Haematol. 1991; 47:119-122. DOI: https:// doi.org/10.1111/j.1600-0609.1991.tb00133.x

12. Wilhelm-Leen $\mathrm{E}$, Montez-Rath ME, Chertow G. Estimating the risk of radiocontrast-associated nephropathy. J Am Soc Nephrol. 2017; 28(2):653-659. DOI:10.1681/ASN.2016010021.

13. Darmon M, Vincent F, Camous L, Canet E, Bonmati C, Braun T, Caillot D, Cornillon J, Dimicoli S, Etienne A, Galicier L, Garnier A, Girault S, Hunault-Berger M, Marolleau JP, Moreau P, Raffoux E, Recher C, Thiebaud A, Thieblemont C, Azoulay E. Tumour lysis syndrome and acute kidney injury in high-risk haematology patients in the rasburicase era. A prospective multicentre study from the Groupe de Recherche en Réanimation Respiratoire et Onco-Hématologique. Br J Haematol, 2013; 162:489-497. DOI:10.1111/bjh.12415

14. Azoulay E, Schellongowski P, Darmon M, Bauer PR, Benoit D, Depuydt P, Divatia JV, Lemiale V, van Vliet M, Meert AP, Mokart D, Pastores SM, Perner A, Pène F, Pickkers P, Puxty KA, Vincent F, Salluh J, Soubani AO, Antonelli M, Staudinger T, von Bergwelt-Baildon M, Soares M. The Intensive Care Medicine research agenda on critically ill oncology and hematology patients. Intensive Care Med. 2017 Sep; 43(9):1366--1382. DOI: 10.1007/s00134-017-4884-z.

15. Wanchoo R, Stotter BR, Bayer RL, Jhaveri KD. Acute kidney injury in hematopoietic stem cell transplantation. Curr Opin Crit Care. 2019; 25(6):531-538. DOI:10.1097/MCC.00000000000000657

16. Shingai N, Morito T, Najima Y, Kobayashi T, Doki N, Kakihana K, Ohashi K, Ando M. Early-onset acute kidney injury is a poor prognostic sign for allogeneic SCT recipients. Bone Marrow Transplant. 2015; 50:1557-1562. DOI: https://doi.org/10.1038/bmt.2015.188
17. Fadia A, Casserly LF, Sanchorawala V, Seldin DC, Wright DG, Skinner M, Dember LM. Incidence and outcome of acute renal failure complicating autologous stem cell transplantation for AL amyloidosis. Kidney Int. 2003; 63(5):1868-1873. DOI: 10.1046/j.1523-1755.2003.00936.x. PMID: 12675865

18. Demoule A, Chevret S, Carlucci A, Kouatchet A, Jaber S, Meziani F, Schmidt M, Schnell D, Clergue C, Aboab J, Rabbat A, Eon B, Guerin C, Georges H, Zuber B, Dellamonica J, Das V, Cousson J, Perez D, Brochard L, Azoulay E. Changing use of noninvasive ventilation in critically ill patients: trends over 15 years in francophone countries. Intensive Care Med. 2016; 42:82-92. DOI: 10.1007/ s00134-015-4087-4.

19. Azoulay $E$, Mokart $D$, Pène $F$, Lambert J, Kouatchet $A$, Mayaux J, Vincent $F$, Nyunga M, Bruneel $F$, Laisne LM, Rabbat A, Lebert C, Perez P, Chaize M, Renault A, Meert A.P, Benoit D, Hamidfar R, Jourdain M, Darmon M, Schlemmer B, Chevret S, Lemiale V. Outcomes of critically ill patients with hematologic malignancies: prospective multicenter data from France and Belgium--a groupe de recherche respiratoire en réanimation onco-hématologique study. J Clin Oncol. 2013 Aug 1; 31(22):2810-2818. DOI: 10.1200/JCO.2012.47.2365.

20. Lahoti A, Kantarjian H, Salahudeen AK, Ravandi F, Cortes JE, Faderl S, O'Brien S, Wierda W, and Mattiuzzi, GN. Predictors and outcome of acute kidney injury in patients with acute myelogenous leukemia or high-risk myelodysplastic syndrome. Cancer. 2010; 116(17):4063-4068. https://doi. org/10.1002/cncr.25306.

21. Park MR, Jeon K, Song J-U, Lim SY, Park SY, Lee JE, Huh W, Kim K, Kim WS, Jung CW, Suh GY. Outcomes in critically ill patients with hematologic malignancies who received renal replacement therapy for acute kidney injury in an intensive care unit. Journal of Critical Care. 2011; 26(1):107. e1-107.e6. DOI: https://doi.org/10.1016/j.jcrc.2010.07.006.

22. Azoulay $E$, Pène $F$, Darmon $M$, Lengliné $E$, Benoit $D$, Soares $M$, Vincent $F$, Bruneel $F$, Perez $P$, Lemiale V, Mokart $D$, Groupe de Recherche Respiratoire en Réanimation Onco-Hématologique (Grrr-OH). Managing critically III hematology patients: Time to think differently. Blood Rev. 2015; 29(6):359-367. DOI:10.1016/j.blre.2015.04.002.

\section{Correspondence to:}

Inês Dionísio Coelho, MD;

Hospital Amato Lusitano, Unidade Local de Saúde de Castelo Branco, EPE, Av.

Pedro Alvares Cabral, 6000-085 Castelo Branco

Email: dcoelho.ines@gmail.com 